\title{
Prevalence, Risk Factors, and Antibiogram of Nontyphoidal Salmonella from Beef in Ambo and Holeta Towns, Oromia Region, Ethiopia
}

\author{
Endrias Zewdu Gebremedhin $\mathbb{D}^{1},{ }^{1}$ Gezahegn Tafesse Soboka, ${ }^{2}$ Bizunesh Mideksa Borana, ${ }^{1}$ \\ Lencho Megersa Marami ${ }^{(D},{ }^{3}$ Edilu Jorga Sarba, ${ }^{1}$ Nega Desalegn Tadese, ${ }^{3}$ \\ and Hirut Abebe Ambecha ${ }^{3}$ \\ ${ }^{1}$ Ambo University, College of Agriculture and Veterinary Sciences, Department of Veterinary Sciences, P.O. Box 19, \\ Ambo, Ethiopia \\ ${ }^{2}$ West Shewa Zone, Ambo District, Ambo, Ethiopia \\ ${ }^{3}$ Ambo University, College of Agriculture and Veterinary Sciences, Department of Veterinary Laboratory Technology, P.O. Box 19, \\ Ambo, Ethiopia
}

Correspondence should be addressed to Endrias Zewdu Gebremedhin; endrias.zewdu@gmail.com

Received 16 December 2020; Revised 2 March 2021; Accepted 17 March 2021; Published 24 March 2021

Academic Editor: Luigi Santacroce

Copyright (c) 2021 Endrias Zewdu Gebremedhin et al. This is an open access article distributed under the Creative Commons Attribution License, which permits unrestricted use, distribution, and reproduction in any medium, provided the original work is properly cited.

\begin{abstract}
Background. Salmonella has been recognized as a major cause of food-borne illness associated with the consumption of food of animal origin. The present cross-sectional study was conducted from December 2017 to May 2018 in Ambo and Holeta towns to assess the prevalence, risk factors, and antimicrobial susceptibility patterns of nontyphoidal Salmonella isolates from raw beef samples from abattoirs, butchers, and restaurants in Ambo and Holeta towns, Oromia region, Ethiopia. Methods. A total of 354 beef samples were collected from abattoirs, butchers, and restaurants. Salmonella isolation and identification were carried out using standard bacteriological methods recommended by the International Organization for Standardization. Antimicrobial susceptibility testing was performed using the disk diffusion method. Besides, a structured questionnaire was used to collect sociodemographic data and potential risk factors for contamination of meat. Chi-square tests and logistic regression were used for data analyses. Results. Of the total 354 meat samples examined, 20 (5.7\%, 95\% confidence interval (CI): 3.5-8.6) were positive for Salmonella. Two serotypes belonging to S. typhimurium (11 isolates) and I:4,5,12: i:- (9 isolates) were identified. The Salmonella detection rate in abattoirs, butchers, and restaurants was 4.2\% (5/118), 8.5\% (10/118), and 4.2\% (5/118), respectively. The antimicrobial susceptibility test showed that $40 \%, 30 \%$, and $20 \%$ of the Salmonella isolates were resistant to azithromycin, amoxicillin, and ceftriaxone, respectively. The odds of Salmonella isolation when meat handlers are illiterate were 7.8 times higher than those when they are educated to the level of secondary and above $(P=0.032)$. Similarly, the likelihood of Salmonella isolation was 6.3 and 7.6 times higher among workers of butcher and restaurants, respectively, who had no training $(P=0.003)$ and no knowledge $(P=0.010)$ on food safety and hygiene. Conclusions. The study showed widespread multidrug-resistant Salmonella isolates in the study areas. Therefore, raw meat consumption and indiscriminate use of antimicrobial drugs should be discouraged. Provision of food safety education for meat handlers and further surveillance of antimicrobial-resistant isolates are suggested.
\end{abstract}

\section{Introduction}

Food-borne bacterial diseases are severe challenges to human and animal health globally. Contaminated food of animal origins such as poultry, pork, beef, and dairy products is often linked to cases of human salmonellosis [1]. Among food-borne pathogens, Salmonella is one of the most important bacteria causing gastroenteritis in humans and animals. It is the leading cause of food-borne diarrhoeal disease and an important public health problem worldwide 
particularly in developing countries [2]. The epidemiology of these diseases has been modified tremendously due to globalization of trade, urbanization, change in consumers' behaviors, increased population, demographic changes, changes in industrial structure, and the capacity of the pathogens to acclimatize to new situations $[3,4]$.

Salmonella, which belongs to the family Enterobacteriaceae, is a Gram-negative, facultatively anaerobic, and non-spore-forming motile rod-shaped bacterium. The bacteria are mostly nonlactose fermenters, oxidase negative, catalase-positive, produce acid and gas from glucose, and utilize citrate as a sole carbon source. Salmonella are classified and identified in 7 subspecies [5]. Currently, the genus Salmonella comprises only two species: S. enterica and S. bongori. The former is further divided into six subspecies, viz., Salmonella enterica subsp. enterica, Salmonella enterica subsp. Arizonae, Salmonella enterica subsp. diarizonae, Salmonella enterica subsp. houtenae, Salmonella enterica subsp. indica, and Salmonella enterica subsp. salamae [5, 6]. Serotypes belonging to Salmonella enterica subsp. enterica are responsible for about $99 \%$ of Salmonella infections in humans and warm-blooded animals, while the other five subspecies and Salmonella bongori are predominantly found in cold-blooded animals and the environment [7]. There are over 2,610 Salmonella serovars [8]. Depending on the host, S. enterica serovar typhimurium (S. typhimurium) can cause diseases ranging from gastroenteritis to life-threatening systemic infection [6].

Salmonella is estimated to cause 93.8 million human gastroenteritis infections and 155, 000 deaths each year worldwide [9]. Although most of these infections cause mild gastroenteritis, life-threatening dispersed infections are common among children, the elderly, and immunocompromised patients [10]. Various animals, including poultry, pigs, cattle, and reptiles, are reservoirs of Salmonella species. A majority of human Salmonella infections are developed after the ingestion of undercooked food of animal origin or contaminated water and vegetables [9].

Salmonella enterica serovars typhimurium and enteritidis have been the major causes of human salmonellosis in developed nations. However, in specific geographical regions, cases of human salmonellosis due to other serovars such as S. Stanley and S. Weltevreden in South-East Asia are common [11]. Salmonella can also spread through the stool of human beings which serves as an important reservoir of Salmonella serovar. Human illness, as well as the spread of Salmonella, could take place when food and water are contaminated with the stool or directly via the feco-oral route [12].

At the butchers' house, meat contamination could occur due to different possible reasons such as storing food in unclean utensils, holding food at a temperature that would allow microbial growth, utilization of poor-quality water, using packaging materials that are not of food-grade quality, a retailing site that had no facilities for waste disposal, and utilization of unclean utensils. Besides, the lack of awareness in basic personal cleanliness and safe food handling of butchers increases the contamination of beef by microorganisms [13]. Meat is a highly perishable food. The rich source of nutrients of fermented meat offers microbes an appropriate milieu to propagate throughout the preparatory step and storage. An upsurge in the demand for beef devoid of the required setup for appropriate healthy handling might lead to the transmission of disease-causing microorganisms from the animals to the consumer [14].

Contamination of meat by Salmonella could occur at abattoir from the excretion of asymptomatic animals, unclean abattoir tools, floor, and workers. The pathogens enter the meat at any stage during slaughtering. Cross-contamination of carcasses and meat products could continue during successive handling, processing, preparation, and delivery [15].

The significance of salmonellosis in the public health sector is a mounting concern day by day worldwide [16]. Salmonellosis frequently follows the ingestion of food of animal origin such as raw meat. Retailing raw meat at butcher shops is extensively accomplished in different towns of Ethiopia including the study areas. Butcher shops are the chief provider of meat of different animals to buyers. Failure to carefully clean work surfaces used to prepare raw meat and other foods in the restaurants can also be a source of Salmonella. Moreover, in Ethiopia, minced beef is usually used for the preparation of a popular traditional Ethiopian dish known as "Kitfo" (minced raw beef mixed with a chili powder-based spice blend and a clarified butter infused with herbs and spices), and most of the time, it is consumed raw or medium-cooked. The renowned custom of raw meat consumption, the presence of Salmonella in minced beef, and the inadequate hygienic standards in food handling indicate the likely occurrence of public health hazards due to Salmonella [17, 18]. This study aimed to estimate the prevalence, antimicrobial susceptibility patterns, and risk factors of nontyphoidal Salmonella in raw beef along the meat chain in Ambo and Holeta towns, Oromia, Ethiopia.

\section{Materials and Methods}

2.1. The Study Area. This study was conducted in Ambo and Holeta towns that are located 115 and $29 \mathrm{kms}$ west of Addis Ababa, respectively. Ambo town is found between $8^{\circ} 56^{\prime} 30^{\prime \prime}$ $\mathrm{N}-8^{\circ} 59^{\prime} 30^{\prime \prime} \mathrm{N}$ latitude and $37^{\circ} 47^{\prime} 30^{\prime \prime} \mathrm{E}-37^{\circ} 55^{\prime} 15^{\prime \prime} \mathrm{E}$ longitude. Holeta is located at approximately $09^{\circ} 03^{\prime}-19.43^{\prime \prime} \mathrm{N}$ latitude and $38^{\circ} 30^{\prime}-25.43^{\prime \prime} \mathrm{E}$ longitude. There are two municipality abattoirs (one in each town) and 92 butcher shops in Ambo $(n=72)$ and Holeta $(n=20)$ towns (Ambo town trade and marketing office, 2017). Stunning, evisceration, and managing of visceral organs were performed in the same room in Ambo as well as Holeta municipality abattoirs.

\footnotetext{
2.2. Study Design and Population. A cross-sectional study was conducted from December 2017 to May 2018. The study populations were cattle slaughtered for human consumption in Ambo and Holeta municipality abattoirs and sold in butcher shops and restaurants operating in the two towns during the study period.
} 
2.3. Sample Size and Sampling Technique. The sample size required was calculated using $17.3 \%$ expected prevalence [19] with a 5\% level of precision and 95\% confidence interval using the formula described as follows [20]:

$$
N=\frac{1.96^{2} \mathrm{P} \exp (1-\mathrm{P} \exp )}{d^{2}},
$$

where, $N=$ sample size, $\mathrm{P}$ exp=expected prevalence, and $d=$ absolute precision. Accordingly, the minimum sample size calculated was 220. Nevertheless, 354 samples were taken deliberately to reduce sample losses during processing and maximize the precision of the study. Of the total meat samples collected, abattoirs, butchers, and restaurants each accounted for 118 samples. The total sample size $(n=354)$ was proportionally distributed to the two towns based on the number of butcher shops that are found in the two towns, i.e., 267 in Ambo and 87 in Holeta towns (1:3 ratios). Systematic random sampling was used to sample cattle in the abattoirs.

\subsection{Study Methodology}

2.4.1. Sample Collection and Transportation. Fresh raw meat samples of cattle origin were collected from different portions of the carcass (neck/brisket, fore rib, flank, and rumpfollowed by pooling) [21] from the abattoir, from displayed meat in the butcher shops, and from restaurants. Samples were kept in separate sterile plastic bags (Seward, England) to prevent spilling and cross-contamination, labeled, and immediately transported to the Ambo University Zoonoses and Food Safety Laboratory in a cooler icebox with ice packs and processed within $4 \mathrm{hrs}$.

\subsubsection{Isolation and Identification of Nontyphoidal Salmo-} nella (NTS). Detection of NTS in the meat samples was performed according to the standard culture method (ISO$6579 ; 2002)$. A $25 \mathrm{~g}$ meat sample was pre-enriched into $225 \mathrm{ml}$ of buffered peptone water (HiMedia, India) and incubated at $37^{\circ} \mathrm{C}$ for $24 \mathrm{hrs}$. Subsequently, $0.1 \mathrm{ml}$ of the preenrichment culture was added to $10 \mathrm{ml}$ of Rappaport-Vassiliadis broth (HiMedia, India) and $1 \mathrm{ml}$ to $10 \mathrm{ml}$ of Mueller Kauffman tetrathionate broth (Microgen, India) and incubated for $24 \mathrm{hrs}$ at $41.5^{\circ} \mathrm{C}$ and $37^{\circ} \mathrm{C}$, respectively. The culture was then streaked on two selective agars, xylose lysine desoxycholate (XLD) (Accumix, Belgium) and brilliant green agar (BGA) (Accumix, Belgium), and incubated at $37^{\circ} \mathrm{C}$ for $24 \mathrm{hrs}$. The formation of red colonies with black centers on XLD and pink colonies on BGA plates was inspected and considered as presumptive Salmonella colonies. For confirmation, up to five presumptive Salmonella colonies from both XLD and BGA agars were selected and streaked onto the surface of predried nutrient agar (HiMedia, India) plates and incubated at $37^{\circ} \mathrm{C}$ for $24 \pm 3 \mathrm{hrs}$. Colonies from nutrient agar were inoculated into the following biochemical tubes for identification: triple sugar iron (TSI) agar (Accumix, Belgium), lysine iron agar (Pronadisa, Spain), Simmon's citrate agar (HiMedia, India), urea agar (HiMedia, India), and indole reaction MIO (motility indole ornithine) medium (Pronadisa, Madrid, Spain) and incubated for 24 or $48 \mathrm{hrs}$ at $37^{\circ} \mathrm{C}$.

2.5. Antimicrobial Susceptibility Test. Nontyphoidal Salmonella isolates were subjected to in vitro antimicrobial susceptibility tests against commonly used antimicrobial drugs using the disk diffusion method following guidelines established by the Clinical and Laboratory Standards Institute [22]. In brief, each isolated bacterial colony from pure fresh culture was transferred into a test tube of $5 \mathrm{ml}$ tryptone soya broth (HiMedia, India) and incubated at $37^{\circ} \mathrm{C}$ for $6 \mathrm{hrs}$. The turbidity of the culture broth was adjusted using a sterile saline solution or by added more isolated colonies to obtain turbidity usually comparable with that of $0.5 \mathrm{McF}$ arland standards (approximately $3 \times 10^{8} \mathrm{CFU}$ per $\mathrm{ml}$ ). The diluted bacterial suspension was transferred to the Mueller-Hinton agar (HiMedia, India) plate using a sterile cotton swab, and the plate was seeded uniformly by rubbing the swab against the entire agar surface followed by $24 \mathrm{hrs}$ incubation. After the inoculums, dried, antimicrobial impregnated disks were applied to the surface of the inoculated plates using sterile forceps. The plate was incubated aerobically at $37^{\circ} \mathrm{C}$ for 24 hrs. Finally, the zone of inhibition was measured using a caliper by including the disk diameter. The susceptible, intermediate, and resistant categories were assigned based on the critical points recommended by the CLSI [22] and according to the manufacturer's leaflet attached to the disks. Standard strains of E. coli ATCC 29522 and ATCC 35218, which are susceptible to all the drugs kindly provided by the Ethiopian Public Health Institute, were used as quality control organisms for the antimicrobial susceptibility test.

2.6. Salmonella Serotyping. Serotyping of Salmonella isolates was done at the Public Health Agency of Canada, World Organization for Animal Health (OIÉ) Reference Laboratory for Salmonellosis, Guelph, Ontario, Canada (report number: UAA160121118R September 12, 2018). In brief, the somatic $(\mathrm{O})$ antigens were determined by slide agglutination tests, and flagella antigens were determined using a microplate agglutination technique [23]. The antigenic formulae of Grimont and Weill [24] were used to identify and assign the serotypes of the isolates.

2.7. Questionnaire Survey and Observations. A structured questionnaire was administered for 269 people working in abattoirs (33), butchers (118), and restaurants (118). The questionnaire survey was administered to assess the potential risk factors for contamination of meat and the knowledge and practice on food hygiene and food safety of workers in abattoirs, butchers, and restaurants. In brief, the questions administered included age in years $(<20,21-29$, 30-39, and $\geq 39$ ), sex (male and female), religion (Orthodox, Protestant, and others), marital status (single, married, and divorced), residential place (urban and rural), level of education (primary, secondary, and tertiary schools), source of meat (abattoirs, butchers, and restaurants), work experience in years, time spent on work per day, information about food 
hygiene and safety (yes/no), training on food safety and hygiene (yes/no), knows food safety and hygiene (yes/no), and practice of food safety and hygiene (yes/no). Moreover, using observational checklists, sanitation of butchers and restaurants, hygiene of slicing materials, cutting boards, and food handlers were rated as poor, fair, and good.

2.8. Variables. Dependent variables: prevalence and antimicrobial susceptibility pattern of Salmonella.

Independent variables: sociodemographic characteristics (sex, age, religion, marital status, educational status, and residence) of food handlers, knowledge about food safety and hygiene (food-borne disease, food contamination, and microorganisms), hygiene and sanitation-related problems of the toilet, water source, waste management, handling practice, and general cleanliness of rooms, dining tables, hand washing basins, and utensils cleaning techniques.

2.9. Data Analysis. The questionnaire and laboratory data were entered into Microsoft Excel Spreadsheet. Data were entered and analyzed using the statistical software STATA version 11.0. Descriptive statistics were used to describe the frequency of Salmonella from different sampling points, antimicrobial susceptibility patterns, and hygienic conditions. The sample level prevalence was calculated as the number of samples positive for Salmonella divided by the total number of samples examined multiplied by 100. Initially, the association between each exposure variable and the presence of Salmonella was assessed using the Chi-square test. Univariable and multivariable logistic regression analyses were done, and crude and adjusted odds ratios with $95 \%$ confidence interval (CI) were calculated for statistical significance tests. Noncollinear variables with $P$ value $<0.25$ in a univariable logistic regression analysis were considered for multivariable analysis to look for a relative effect on the outcome variable by controlling other possible confounding factors. The binomial exact method was used to calculate the $95 \%$ confidence interval (CI) of the prevalence estimates. $P$ value $<0.05$ was considered statistically significant.

\section{Results}

3.1. Prevalence of Salmonella. Out of 354 samples tested, the prevalence of nontyphoidal Salmonella was found to be $5.7 \%$ $(95 \% \mathrm{CI}=3.5-8.6 \%)$. The prevalence of Salmonella contamination in Ambo and Holeta towns was 7.5\% (95\% $\mathrm{CI}=4.6-11.3 \%$ ) and $0 \%$, respectively (Table 1 ).

3.2. Salmonella Serotype Distribution. Of the 20 isolates subjected to the serotyping, all isolates were confirmed to be Salmonella. Overall, 2 different Salmonella serotypes were recovered, the predominant serotype being S. typhimurium followed by I:4,5,12:i:- (Table 2).

3.3. Antimicrobial Susceptibility of Salmonella. Salmonella isolates were subjected to an antimicrobial susceptibility test using 15 selected antimicrobial drugs (Table 3). The isolated strains were $100 \%, 95 \%, 95 \%$, and $80 \%$ susceptible to cotrimoxazole, chloramphenicol, norfloxacin, and nitrofurantoin, respectively. In other cases, $40 \%, 30 \%$, and $25 \%$ of the Salmonella isolates were resistant to azithromycin, amoxicillin, and nalidixic acid, respectively (Table 3 ).

Salmonella isolates showed sixteen different antimicrobial resistance patterns (Table 4). Out of 20 Salmonella isolates, $6(30 \%)$ developed resistance to three or more classes of antimicrobial drugs, i.e., multidrug resistance (MDR). One isolate (5\%) had developed resistance to five classes of antimicrobials. Nine isolates (45\%) of Salmonella isolates were resistant to at least two classes of antimicrobial drugs.

3.4. Sociodemographic Characteristics of the Workers. The sociodemographic information of abattoir, meat retailer, and restaurant workers interviewed in Ambo and Holeta towns showed that most of the respondents from abattoirs (66.7\%), retailer shops (51.7\%), and restaurants (50.9\%) were between the ages of 21 and 29 years. A majority (94.9\%) of the workers in the restaurants were females. On the other hand, the majority of the workers in abattoirs (51.5\%), butcher shops (67.8\%), and restaurants (56.8\%) had primary school level education (Table 5).

3.5. Knowledge and Practice on Food Hygiene and Food Safety. Generally, $93.9 \%, 72.0 \%$, and $83.1 \%$ of the respondents working in abattoirs, butchers, and restaurants have information about food hygiene and safety, respectively. About, $66.7 \%, 44.9 \%$, and $66.1 \%$ of the respondents working in abattoirs, butchers, and restaurants, respectively, know about food hygiene and safety, while $45.5 \%, 42.4 \%$, and $68.6 \%$ of them working in the respective places had a good practice on food safety and hygiene (Table 6). During our observation, the gates of the Ambo town abattoir were always open without any restriction to personal movement to go inside and out of the abattoir, and the hygiene of the surroundings and its interior of the abattoir were poor. Moreover, $51.7 \%$ of the butcher shop workers and $38.1 \%$ of the restaurant workers did not cover their hair while performing their duties at their working place. It has been also observed that the retailer operators retailing meat wipe their hands, cutting board, and weighing balance surfaces with dirty reusable clothes. The same piece of cloth was used throughout the day for drying hands, knives, and chopping boards. Most (56.8\%) of the butcher workers did not wash their hands after handling the money. Besides, observational findings showed the absence of washing basins and first aid kits in almost all butchers and restaurants.

3.6. Potential Risk Factors for Meat Contamination. Univariable logistic regression analysis revealed that religion $(P=0.018)$, education $(P=0.028)$, training on food safety and hygiene $(P=0.001)$, and time spent on the work per day $(P=0.008)$ were significantly associated with the isolation rate of Salmonella. At the same time, knowledge of food safety and hygiene $(P=0.004)$, sanitation of butcher/ 
TABle 1: Prevalence of Salmonella in abattoirs, butchers, and restaurants of Ambo and Holeta towns.

\begin{tabular}{lcccccc}
\hline \multirow{2}{*}{ Sources of meat } & \multicolumn{2}{c}{ Ambo town } & \multicolumn{2}{c}{ Holeta town } & \multicolumn{2}{c}{ Total } \\
& No. examined & Salmonella positive (\%) & No. examined & Salmonella positive (\%) & No. examined & Prevalence (\%) \\
\hline Abattoirs & 89 & $5(5.6)$ & 29 & $0(0)$ & 118 & 4.2 \\
Butchers & 89 & $10(11.2)$ & 29 & $0(0)$ & 118 & 8.5 \\
Restaurants & 89 & $5(5.6)$ & 29 & $0(0)$ & 118 & 4.2 \\
Total & 267 & $20(7.5)$ & 87 & $0(0)$ & 354 \\
\hline
\end{tabular}

TABLE 2: Salmonella serotypes distribution and site of isolation.

\begin{tabular}{lcccc}
\hline Serotypes & & \multicolumn{2}{c}{ Site of isolation } & \\
& Abattoirs & Butchers & Restaurants & Total \\
\hline S. typhimurium & 3 & 3 & 5 & 11 \\
I:4,5,12:i:- & 2 & 7 & 0 & 9 \\
Total & 5 & 10 & 5 & 20 \\
\hline
\end{tabular}

TABle 3: Antimicrobial susceptibility test of Salmonella isolates from meat samples of Ambo and Holeta towns, central Ethiopia.

\begin{tabular}{|c|c|c|c|c|}
\hline \multirow{2}{*}{$\begin{array}{l}\text { Antimicrobial } \\
\text { class }\end{array}$} & \multirow{2}{*}{$\begin{array}{l}\text { Antimicrobial discs and } \\
\text { concentration }\end{array}$} & \multicolumn{3}{|c|}{ Salmonella isolates $(n=20)$} \\
\hline & & $\begin{array}{c}\text { No. of susceptible isolates } \\
(\%)\end{array}$ & $\begin{array}{c}\text { No. of. intermediate isolates } \\
(\%)\end{array}$ & $\begin{array}{c}\text { No. of. resistant isolates } \\
(\%)\end{array}$ \\
\hline \multirow{2}{*}{ Aminoglycosides } & Amikacin $(30 \mu \mathrm{g})$ & $15(75.0)$ & $5(25.0)$ & $0(0.0)$ \\
\hline & Gentamicin $(10 \mu \mathrm{g})$ & $16(80.0)$ & $2(10.0)$ & $2(10.0)$ \\
\hline \multirow{3}{*}{ Cephems } & Cefotaxime $(30 \mu \mathrm{g})$ & $17(85.0)$ & $1(5.0)$ & $2(10.0)$ \\
\hline & Ceftazidime $(30 \mu \mathrm{g})$ & $16(80.0)$ & $0(0.0)$ & $4(20.0)$ \\
\hline & Ceftriaxone $(5 \mu \mathrm{g})$ & $15(75.0)$ & $1(5.0)$ & $4(20.0)$ \\
\hline Macrolide & Azithromycin $(30 \mu \mathrm{g})$ & $3(15.0)$ & $9(45.0)$ & $8(40.0)$ \\
\hline Nitrofurans & Nitrofurantoin $(300 \mu \mathrm{g})$ & $16(80.0)$ & $1(5.0)$ & $3(15.0)$ \\
\hline \multirow[t]{2}{*}{ Phenicols } & Chloramphenicol $(30 \mu \mathrm{g})$ & $19(95.0)$ & $0(0.0)$ & $1(5.0)$ \\
\hline & Ciprofloxacin $(5 \mu \mathrm{g})$ & $12(60.0)$ & $8(40.0)$ & $0(0.0)$ \\
\hline \multirow[t]{2}{*}{ Quinolones } & Nalidixic acid $(30 \mu \mathrm{g})$ & $15(75.0)$ & $0(0.0)$ & $5(25.0)$ \\
\hline & Norfloxacin $(10 \mu \mathrm{g})$ & $19(95.0)$ & $1(5.0)$ & $0(0.0)$ \\
\hline Sulphonamides & Cotrimoxazole $(25 \mu \mathrm{g})$ & $20(100.0)$ & $0(0.0)$ & $0(0.0)$ \\
\hline Tetracycline & Tetracycline $(30 \mu \mathrm{g})$ & $17(85.0)$ & $0(0.0)$ & $3(15.0)$ \\
\hline \multirow{2}{*}{$\beta$-Lactams } & Ampicillin $(10 \mu \mathrm{g})$ & $18(90.0)$ & $1(5.0)$ & $1(5.0)$ \\
\hline & Amoxicillin $(25 \mu \mathrm{g})$ & $5(25.0)$ & $9(45.0)$ & $6(30.0)$ \\
\hline
\end{tabular}

TABle 4: Multidrug resistance patterns in Salmonella isolated from meat samples in Ambo and Holeta towns.

\begin{tabular}{|c|c|c|}
\hline Number & Antimicrobial resistance pattern & No. of resistant isolates (\%) \\
\hline \multirow{10}{*}{ Two } & AMX, AZM & $3(9.7)$ \\
\hline & AZM, TET & $3(9.7)$ \\
\hline & NA,CTR & $3(9.7)$ \\
\hline & NA, CAZ & $3(9.7)$ \\
\hline & AMX, TET & $2(6.5)$ \\
\hline & AMX, NA & $2(6.5)$ \\
\hline & AMX, CTR & $2(6.5)$ \\
\hline & AZM, GEN & $2(6.5)$ \\
\hline & AMX, AZM, NIT & $2(6.5)$ \\
\hline & AMX, AZM, TET & $2(6.5)$ \\
\hline \multirow{3}{*}{ Three } & AZM, NIT, TET & $2(6.5)$ \\
\hline & AMX, CXT, NA & $1(3.2)$ \\
\hline & AZM, CXT, NA & $1(3.2)$ \\
\hline \multirow{2}{*}{ Four } & AMX, AZM, NIT, TET & $1(3.2)$ \\
\hline & AZM, CHL, NIT, TET & $1(3.2)$ \\
\hline Five & AMX, AZM, GEN, NA, TET & $1(3.2)$ \\
\hline Total & & $31(100 \%)$ \\
\hline
\end{tabular}

AZM: azithromycin; AMX: amoxicillin; CTR: ceftriaxone; TET: tetracycline; NA: nalidixic acid; CXT: cefotaxime; CHL: chloramphenicol; NIT: nitrofurantoin; GEN: gentamycin. 
TABLE 5: Sociodemographic characteristics of abattoir, butcher, and restaurant workers in Ambo and Holeta towns.

\begin{tabular}{|c|c|c|c|c|c|c|c|}
\hline \multirow{2}{*}{ Variables } & \multirow{2}{*}{ Categories } & \multicolumn{2}{|c|}{ Abattoir workers } & \multicolumn{2}{|c|}{ Butcher workers } & \multicolumn{2}{|c|}{ Restaurant workers } \\
\hline & & Freq. & $\%$ & Freq. & $\%$ & Freq. & $\%$ \\
\hline \multirow{4}{*}{ Age in years } & Less than 20 & 7 & 21.2 & 14 & 11.9 & 34 & 28.8 \\
\hline & $21-29$ & 22 & 66.7 & 61 & 51.7 & 60 & 50.9 \\
\hline & $30-38$ & 2 & 6.1 & 31 & 26.3 & 24 & 20.3 \\
\hline & $\geq 39$ & 2 & 6.1 & 12 & 10.2 & 0 & 0 \\
\hline \multirow{2}{*}{ Sex } & Male & 33 & 100 & 118 & 100 & 6 & 5.1 \\
\hline & Female & 0 & 0 & 0 & 0 & 112 & 94.9 \\
\hline \multirow{3}{*}{ Religion } & Orthodox & 21 & 63. & 111 & 94.1 & 75 & 63.6 \\
\hline & Protestant & 10 & 30.30 & 7 & 5.9 & 40 & 33.9 \\
\hline & Others & 2 & 6.1 & 0 & 0 & 3 & 2.5 \\
\hline \multirow{3}{*}{ Marital status } & Single & 15 & 45.5 & 45 & 38.1 & 61 & 51.7 \\
\hline & Married & 18 & 54.6 & 73 & 61.9 & 54 & 45.8 \\
\hline & Divorced & 0 & 0 & 0 & 0 & 3 & 2.5 \\
\hline \multirow{2}{*}{ Residence } & Urban & 26 & 78.8 & 77 & 65.3 & 53 & 44.9 \\
\hline & Rural & 7 & 21.2 & 41 & 34.8 & 65 & 55.1 \\
\hline \multirow{4}{*}{ Education } & Illiterate & 3 & 9.1 & 9 & 7.6 & 18 & 15.3 \\
\hline & Primary (1-8) & 17 & 51.5 & 67 & 56.8 & 80 & 67.8 \\
\hline & Secondary (9-12) & 6 & 18.2 & 34 & 28.8 & 20 & 17.0 \\
\hline & Tertiary & 7 & 21.2 & 8 & 6.8 & 0 & 0 \\
\hline
\end{tabular}

Freq. $=$ frequency.

TABLE 6: Knowledge and practice on food hygiene and food safety.

\begin{tabular}{|c|c|c|c|c|c|c|c|}
\hline \multirow[t]{2}{*}{ Variables } & \multirow[t]{2}{*}{ Categories } & \multicolumn{2}{|c|}{ Abattoir workers } & \multicolumn{2}{|c|}{ Butcher workers } & \multicolumn{2}{|c|}{$\begin{array}{c}\text { Restaurants } \\
\text { workers }\end{array}$} \\
\hline & & Freq. & $\%$ & Freq. & $\%$ & Freq. & $\%$ \\
\hline \multirow{2}{*}{ Have food hygiene and safety information } & Yes & 31 & 93.9 & 85 & 72.0 & 98 & 83.1 \\
\hline & No & 2 & 6.1 & 33 & 28.0 & 20 & 16.9 \\
\hline \multirow{2}{*}{ Has training on food safety and hygiene } & Yes & 5 & 15.2 & 27 & 22.9 & 9 & 7.6 \\
\hline & No & 28 & 84.9 & 91 & 77.1 & 110 & 92.4 \\
\hline \multirow{2}{*}{ Knows food safety and hygiene } & Yes & 22 & 66.7 & 53 & 44.9 & 78 & 66.1 \\
\hline & No & 11 & 33.3 & 65 & 55.1 & 40 & 33.9 \\
\hline \multirow{2}{*}{ Practice food safety and hygiene } & Yes & 15 & 45.5 & 50 & 42.4 & 81 & 68.6 \\
\hline & No & 18 & 54.6 & 68 & 57.6 & 37 & 31.3 \\
\hline
\end{tabular}

restaurants $(P=0.009)$, hygiene of slicing materials $(P=0.003)$, and hygiene of food handlers $(P=0.002)$ were also significantly associated with the isolation rate of Salmonella. In this study, the univariable analysis showed that the isolation of Salmonella was 7 times higher in food establishments where food handlers are illiterate than the others $(\mathrm{OR}=6.8,95 \% \mathrm{CI}=1.2-37.7, P=0.028)$. In establishments where the food handlers had no previous training on food safety and hygiene, isolation of Salmonella was 6 times higher than those who had the training $(\mathrm{OR}=6.03$, $95 \% \mathrm{CI}=2.0-17.9, P=0.001)$. The duration of time spent in the handling of meat per day had also a role in the isolation of Salmonella. The likelihood of isolation was nearly 8 times higher in an establishment where the food handlers worked more than 13 hrs per day as compared to those who spent $8-12 \mathrm{hrs}$ per day $(\mathrm{OR}=7.7 ; 95 \% \mathrm{CI}=1.7-35.0, P=0.008)$. The knowledge of the food handlers on food safety and hygiene also had an impact on the isolation rate of Salmonella. In this study, the probability of isolation of Salmonella was 9 times higher in those establishments where food handlers had no knowledge of food safety and hygiene than those who knew $(\mathrm{OR}=9.11,95 \%$ CI: 2.0-41.4, $P=0.004)$. The odds of isolating Salmonella was also nearly 6 times higher in butchers and restaurants where the sanitation was poor $(\mathrm{OR}=5.8,95 \% \mathrm{CI}=1.6-21.8, P=0.009)$. The poor hygiene of the slicing material and food handlers were 8 and 10 times more likely to yield isolation of Salmonella when compared with fair and good hygiene $(\mathrm{OR}=7.9,95 \% \mathrm{CI}=2.0-31.8, P=0.003$ and $\mathrm{OR}=10,95 \%$ $\mathrm{CI}=2.5-46.1, P=0.002$ ), respectively (Table 7 ).

All the independent variables investigated were noncollinear with each other except sex of the respondent versus the meat source $(r=0.9)$, the practice of food safety and hygiene of the meat handlers versus their knowledge on food safety and hygiene $(r=0.9)$, and hygiene of the food handlers versus the hygiene of cutting board $(r=0.8)$. Meat source, knowledge of the workers, and hygiene of the food handlers were selected for entry into the multivariable model.

In the univariable logistic regression analysis, source, age, religion, education, food safety training, time spent on 
TABLE 7: Univariable logistic regression analysis of factors associated with meat contamination with Salmonella in butcher and restaurants of Ambo and Holeta towns.

\begin{tabular}{|c|c|c|c|c|c|c|c|}
\hline Variables & Categories & Tested & Pos. & $\%$ & OR & $95 \% \mathrm{CI}$ & $P$ value \\
\hline \multirow{2}{*}{ Source } & Restaurants & 118 & 5 & 4.2 & 1.0 & & \\
\hline & Butchers & 118 & 10 & 8.5 & 2.0 & $0.7-6.3$ & 0.110 \\
\hline \multirow{2}{*}{ Sex } & Female & 124 & 5 & 4.5 & 1.0 & & \\
\hline & Male & 112 & 10 & 8.1 & 1.9 & $0.6-5.7$ & 0.264 \\
\hline \multirow{3}{*}{ Age in years } & $\leq 24$ & 89 & 2 & 2.1 & 1.0 & & \\
\hline & $25-30$ & 96 & 9 & 9.4 & 3.7 & $0.7-21.0$ & 0.139 \\
\hline & $\geq 31$ & 51 & 4 & 7.8 & 4.5 & $0.9-21.4$ & 0.059 \\
\hline \multirow{2}{*}{ Marital status } & Single & 106 & 6 & 5.7 & 1.0 & & \\
\hline & Married and divorced & 130 & 9 & 6.9 & 1.2 & $0.4-3.3$ & 0.693 \\
\hline \multirow{2}{*}{ Residence } & Rural & 106 & 6 & 5.7 & 1.0 & & \\
\hline & Urban & 130 & 9 & 6.9 & 1.2 & $0.4-3.3$ & 0.693 \\
\hline \multirow{2}{*}{ Religion } & Orthodox & 186 & 8 & 4.3 & 1.0 & & \\
\hline & Protestant & 50 & 7 & 14 & 3.6 & $1.3-10.5$ & 0.018 \\
\hline \multirow{3}{*}{ Education } & Secondary and above & 27 & 5 & 18.5 & 1.0 & & \\
\hline & Primary & 147 & 8 & 5.4 & 1.7 & $0.4-8.4$ & 0.498 \\
\hline & Illiterate & 62 & 2 & 3.2 & 6.8 & $1.2-37.7$ & 0.028 \\
\hline \multirow{3}{*}{ Experience in years } & $<1$ & 51 & 2 & 3.9 & 1.0 & & \\
\hline & $1-3$ & 133 & 9 & 6.8 & 1.8 & $0.4-8.5$ & 0.472 \\
\hline & $\geq 4$ & 52 & 4 & 7.7 & 2.0 & $0.4-11.7$ & 0.422 \\
\hline \multirow{2}{*}{ Has information about food safety and hygiene } & No & 183 & 13 & 7.1 & 1.0 & & \\
\hline & Yes & 53 & 2 & 3.8 & 1.9 & $0.4-8.9$ & 0.390 \\
\hline \multirow{2}{*}{ As training on food safety and hygiene } & Yes & 35 & 7 & 20 & 1.0 & & \\
\hline & No & 201 & 8 & 4.0 & 6.0 & $2.0-17.9$ & 0.001 \\
\hline \multirow{2}{*}{ Time spend on the work per day } & $8-12$ hrs & 122 & 2 & 1.6 & 1.0 & & \\
\hline & $\geq 13 \mathrm{hrs}$ & 114 & 13 & 11.4 & 7.7 & $1.7-35.0$ & 0.008 \\
\hline \multirow{2}{*}{ Knows food safety and hygiene } & Yes & 131 & 2 & 1.5 & 1.0 & & \\
\hline & No & 105 & 13 & 12.4 & 9.1 & $2.0-41.4$ & 0.004 \\
\hline \multirow{2}{*}{ Practice food safety and hygiene } & Yes & 131 & 7 & 5.3 & 1.0 & & \\
\hline & No & 105 & 8 & 7.6 & 1.5 & $0.5-4.2$ & 0.479 \\
\hline \multirow{3}{*}{ Sanitation of butcher/restaurants } & Fair & 26 & 5 & 19.2 & 1.0 & & \\
\hline & Good & 127 & 5 & 3.9 & 1.6 & $0.4-5.6$ & 0.491 \\
\hline & Poor & 83 & 5 & 4.8 & 5.8 & $1.6-21.8$ & 0.009 \\
\hline \multirow{3}{*}{ Hygiene of slicing material } & Fair & 14 & 4 & 28.6 & 1.0 & & \\
\hline & Good & 146 & 7 & 4.8 & 1.1 & $0.3-3.9$ & 0.879 \\
\hline & Poor & 76 & 4 & 5.3 & 7.94 & $2.0-31.8$ & 0.003 \\
\hline \multirow{3}{*}{ Hygiene of cutting board } & Good & 18 & 3 & 16.7 & 1.0 & & \\
\hline & Fair & 127 & 7 & 5.5 & 1.0 & $0.3-3.3$ & 0.996 \\
\hline & Poor & 91 & 5 & 5.5 & 4.4 & $0.7-15.9$ & 0.114 \\
\hline \multirow{3}{*}{ Hygiene of food handlers } & Good & 24 & 6 & 25.0 & 1.0 & & \\
\hline & Fair & 114 & 6 & 5.3 & 1.8 & $0.4-7.2$ & 0.433 \\
\hline & Poor & 98 & 3 & 3.1 & 10 & $2.5-46.1$ & 0.002 \\
\hline \multirow{2}{*}{ Refrigerator using } & Yes & 222 & 14 & 6.4 & 1.0 & & \\
\hline & No & 14 & 1 & 7.1 & 1.1 & $0.1-9.4$ & 0.901 \\
\hline \multirow{2}{*}{ Presence of insect } & No & 187 & 12 & 6.4 & 1.0 & & \\
\hline & Yes & 49 & 3 & 6.1 & 1.1 & $0.3-3.9$ & 0.940 \\
\hline \multirow{2}{*}{ Presence of rodents } & Yes & 53 & 2 & 1.9 & 1.0 & & \\
\hline & No & 183 & 13 & 7.1 & 1.9 & $0.4-8.9$ & 0.390 \\
\hline
\end{tabular}

the work per day, food safety and hygiene knowledge, sanitation of the butcher/restaurants, hygiene of the slicing materials, and hygiene of the food handlers were associated with the isolation rate of Salmonella at a $P$ value of $\leq 0.25$. On the other hand, the multivariable logistic regression analysis revealed that the age of the meat handlers, the educational background of the food handlers, the food handlers training on food safety and hygiene, and food safety and hygiene knowledge were significantly and independently associated with the isolation of Salmonella, $P<0.05$. The odds of isolating Salmonella was 8 times $(P=0.049)$ higher in bluchers and restaurants where the workers did not attend formal education compared to those who attended secondary school and above. Likewise, isolation of Salmonella 
was 10.5 times higher in butchers and restaurants where the food handlers had no food safety and hygiene knowledge $(P=0.006)$. The likelihood of isolating Salmonella was 5.7 times higher $(P=0.008)$ where the food handlers had no previous training on food safety and hygiene than those who had the training (Table 8).

Model selection to identify the best-fitting model showed the level of education of the workers, training of the workers on food safety, and knowledge on food safety and sanitation to be the independent predictors of Salmonella isolation (Table 9). The data fitted well the model (Hosmer-Lemeshow Chi-square $=5.85, P=0.3210$, area under curve $(\mathrm{ROC})=0.8730)$.

\section{Discussion}

The present study was conducted to estimate the prevalence of Salmonella and assess associated risk factors for contamination and antimicrobial susceptibility profile of isolates from meat samples collected from abattoirs, butchers, and restaurants in Ambo and Holeta towns.

Reports from other parts of the world showed varying prevalence, the serotype of Salmonella, and antimicrobial resistance levels from raw beef. In developed countries, a prevalence of $0.6 \%$ to $4.2 \%$ Salmonella serotypes in raw beef was reported in Germany [25], Canada [26], and the USA $[27,28]$. Relatively higher contamination rates of Salmonella in retail beef samples were reported from Malaysia (9.6\%) [29], China (17\%) [30], and Egypt (8.8\%) [31]. In most of these reports, they also found moderate to high MDR Salmonella isolates. The difference in the occurrence of Salmonella in beef among countries could be partly due to the variation in the hygienic meat handling and processing practice, the types of samples analyzed (whole carcass or steaks or frozen or fresh), and the sensitivity diagnostic test applied.

The overall prevalence of Salmonella in the current study towns (5.7\%) was higher than that reported from Addis Ababa abattoir enterprise [32], Hawassa [33], Addis Ababa University student's lunchroom [34], and Gondar University [35], which reported a prevalence of $4.6 \%, 4 \%, 3.5 \%$, and $3.1 \%$, respectively. A much lower than the present study was also reported from Jimma (1.2\%) [35] and central Ethiopia (2.3\%) [36].

However, the prevalence of Salmonella in this study was lower than the prevalence reported in other studies in Ethiopia such as $10.8 \%$ in Jimma [37], 12.9\% in Addis Ababa [38], 12.5\% in Wolaita Sodo [39], 14\% in central Ethiopia [40], 15\% in Bahir Dar [41], 17.3\% in Gondar town [19], and $17.5 \%$ in Arba Minch [42]. The prevalence from Gondar (5.5\%) [43] and Jimma (4.4\%) [44] is in line with this study. The probable reason for the variations in the prevalence of Salmonella between studies could be due to differences in sample size, sampling techniques, laboratory procedures, study areas, seasons, and hygienic conditions employed [45].

Although there is a difference in prevalence, the trend of an increase in Salmonella isolation in the current study from abattoirs $(4.24 \%)$ and butchers $(8.47 \%)$ was similar to the results from Addis Ababa abattoir (26.3\%) and butchers
(32.4\%) [38]. The significant variation in the prevalence between the abattoirs and the butcher shops might be due to the risk of direct carcass cross-contamination during loading and transportation of the carcass using a single car and unhygienic handling of meat at butcher shops (personal observation). Abattoir workers carry the meat on their back or chest, hold, and support it using their two hands. Therefore, the unhygienic handling practice of meat and direct contact between contaminated clothes of loader and carcass might be the cause for the higher prevalence of Salmonella from the butcher shops. Furthermore, all the butcher shops are located along the major roadsides, and meat displayed for sale was not covered thus exposing the meat to contamination by flies and dust. On the contrary, the relatively lower prevalence of Salmonella in meat samples from restaurants ("Kitfo") might be because meat for "Kitfo" preparation was taken from the softer part following the removal of some layer of meat which might have been exposed to contamination.

S. typhimurium, the dominant serotypes isolated in the current study, is one of the common causes of nontyphoidal salmonellosis in people [46]. The three top-ranked Salmonella serotypes commonly identified from cattle slaughtered in Addis Ababa are S. mishmarhaemek, S. typhimurium, and S. enteritidis [47]. In a different study carried out in northern Ethiopia from slaughtered cattle, S. typhimurium and S. newport were the two dominant serotypes isolated [41]. The other serotype detected in this study was S. enterica serotype 1,4,5,12: i:-, a monophasic variant of Salmonella typhimurium, which has also been isolated previously from slaughtered cattle carcass swabs, cecal contents, and slaughterhouse environment from southern Ethiopia [33]. This serotype is emerging in recent years and has become one of the most common serotypes isolated from humans and swine enteric salmonellosis [48]. Even though there is limited diversity in the serotypes in the current study, it is believed that this study adds to the global knowledge on the occurrence, risk factors, and antimicrobial resistance particularly concerning the emerging Salmonella enterica serotype $1,4,5,12$ : i:-.

Antimicrobial resistance is an emerging global problem in human and veterinary medicine in both developed and developing nations. The important factor for the production of bacterial resistance is the expanded utilization of antimicrobial agents in food animal production and humans [49]. In the present study, all Salmonella isolates were highly susceptible to cotrimoxazole, chloramphenicol, norfloxacin, and nitrofurantoin. This is in line with the findings from food handlers in Arba Minch University student's lunchroom [50], food handlers of Gondar University student lunchroom [19], dairy farms in Modjo [51], and from milk samples from Addis Ababa [52]. In this study, all the Salmonella isolates were susceptible to cotrimoxazole unlike the other reports from different parts of Ethiopia where resistance to cotrimoxazole ranging from 34.5 to $100 \%$ has been reported $[42,53,54]$.

The current study revealed that Salmonella isolates were resistant to azithromycin (40\%), amoxicillin (30\%), ceftriaxone (20\%), ceftazidime (20\%), nalidixic acid (25\%), and 
TABLE 8: Multivariable logistic regression analysis of factors associated with the contamination of meat with Salmonella in Ambo and Holeta towns, central Ethiopia.

\begin{tabular}{|c|c|c|c|c|}
\hline Variables & Categories & AOR & $95 \% \mathrm{CI}$ & $P$ value \\
\hline \multirow{3}{*}{ Age in years } & $\leq 24$ & 1.0 & & \\
\hline & $25-30$ & 2.8 & $0.4-21.1$ & 0.315 \\
\hline & $\geq 31$ & 6.5 & $1.1-39.3$ & 0.040 \\
\hline \multirow{3}{*}{ Education } & Secondary and above & 1.0 & & \\
\hline & Primary & 1.68 & $0.3-10.5$ & 0.578 \\
\hline & Illiterate & 8.0 & $1.0-64.3$ & 0.049 \\
\hline \multirow{2}{*}{ Has training on food safety and hygiene } & Yes & 1.0 & & \\
\hline & No & 5.7 & $1.6-20.8$ & 0.008 \\
\hline \multirow{2}{*}{ Knows food safety and hygiene } & Yes & 1.0 & & \\
\hline & No & 10.52 & $1.9-57.2$ & 0.006 \\
\hline \multirow{3}{*}{ Sanitation of butcher/restaurants } & Fair & 1.0 & & \\
\hline & Good & 3.69 & $0.9-15.8$ & 0.078 \\
\hline & Poor & 4.16 & $0.8-22.3$ & 0.094 \\
\hline
\end{tabular}

$\mathrm{AOR}=$ adjusted odds ratio; $\mathrm{CI}=$ confidence interval. The values in bold are statistically significant.

TABLE 9: Best-fitting model for predictors of Salmonella isolation in butchers and restaurants of Ambo and Holeta towns, central Ethiopia.

\begin{tabular}{|c|c|c|c|c|}
\hline Variables & Categories & AOR & $95 \% \mathrm{CI}$ & $P$ value \\
\hline \multirow{3}{*}{ Education } & Secondary and above & 1.0 & & \\
\hline & Primary & 1.6 & $0.3-8.3$ & 0.582 \\
\hline & Illiterate & 7.8 & $1.2-51.2$ & 0.032 \\
\hline \multirow{2}{*}{ Has training on food safety and hygiene } & Yes & 1.0 & & \\
\hline & No & 6.3 & $1.9-21.2$ & 0.003 \\
\hline \multirow{2}{*}{ Knows food safety and hygiene } & Yes & 1.0 & & \\
\hline & No & 7.6 & $1.6-35.9$ & 0.010 \\
\hline
\end{tabular}

The values in bold are statistically significant.

tetracycline $(15 \%)$. In line with this, the first failure of azithromycin therapy was reported in 2010 in a patient with invasive Salmonella infection [55]. The resistance rate of the isolates to amoxicillin in this study was less than the $100 \%$ resistance reported in the Nekemte Referral Hospital [56], and the $69.2 \%$ reported in Gondar University [19]. Similarly, much lower resistance to ampicillin (5\%) and tetracycline (15\%) was recorded in the current study as compared to previous investigations where resistances ranging from $82.3-100 \%$ for ampicillin $[32,37,57]$ and $47.4 \%$ for tetracycline [37] have been reported. The $22 \%$ resistance rate to nalidixic acid in this study was similar to that of Lamboro et al. [37].

In the current study, both serotypes identified were resistant to different antimicrobials. Of the 20 isolates, 6 (30\%) were resistant to three or more classes of antimicrobial drugs, while 9 isolates (45\%) were resistant to at least two classes of drugs. The percentage of the MDR isolates in the current study is less than the previous reports, which ranges from $75.5 \%$ to $84.6 \% \operatorname{MDR}[19,32,53]$. Since most of these drugs are also commonly used in human medicine in Ethiopia, this resistance to antimicrobials is highly significant. For instance, ciprofloxacin and ceftriaxone are used for the treatment of bacillary dysentery; chloramphenicol, trimethoprim/sulfamethoxazole, and ciprofloxacin are used against gastroenteritis; the latter two drugs are also used to treat cholera; amoxicillin, gentamycin, and ceftriaxone are used to treat pneumonia; trimethoprim/sulfamethoxazole and amoxicillin are used against sinusitis [58]. Moreover, it has been observed that prescriptions are usually made without prior isolation and performing drug susceptibility tests for infectious agents in the study areas. Furthermore, the possible lateral transmission of resistance traits and other virulence factors or plasmids from Salmonella to different microorganisms, inside the human gut, may exist. Resistance traits in Salmonella can be hereditarily decided and may include chromosomal mutations or plasmid-mediated and might be exchanged with other Enterobacteriaceae species [59]. There is a lack of advocacy and monitoring of antimicrobial drug utilization at all levels in Ethiopia. Additionally, underdose or prophylactic usage of the drugs in food animals may produce antimicrobial resistance genes in Salmonella as well as other potential human and animal pathogens.

The high antimicrobial resistance rates observed in this study for some drugs might have been due to the natural process or could be because of the uncontrolled accessibility of the antimicrobial agents in drug vendors, which prompts abuse and greater selection pressure for resistant strains. It could also be due to the deficiency or nonappearance of antimicrobial resistance observation programs $[60,61]$. The presence of antimicrobial resistance adversely affects human health by causing severe illness that is more difficult and expensive to treat. This is because resistant infections are more severe and those patients are more likely to be hospitalized, and treatment in such cases is less effective [49]. 
The study noted that $79.6 \%$ of the respondent had information about food safety from nonformal ways such as mass media, friends, and parents. The previous study indicated that proper training, education, and monitoring of the workers could address the limitation of the implementation of personal hygienic practices [62]. Despite most respondents in the present study (92.6\%) did not receive any formal training regarding hygiene and sanitation of meat handling neither prior nor after employment, $66.7 \%$ of the abattoirs, $73.7 \%$ of butchers, and $50.8 \%$ of restaurants workers knew contaminated meat could cause bacterial diseases (Table 7). A study conducted in Malaysia reported that $73.4 \%$ of food handlers had acceptable knowledge of food-borne pathogens [63]. In this study, the knowledge of the respondent about food hygiene and safety as well as the food-borne diseases causing microorganisms is $62.8 \%$, which is less than the report mentioned in Malaysia. Since meat handlers can serve as vehicles for cross-contamination and the spread of food-borne pathogens, they need to know the importance of proper meat handling, hand washing, and other important hygienic procedures [63]. With this regard, it has been well documented that training enhances food handlers' awareness of food-borne diseases and could enable them to better understand and fulfill their responsibilities and exercise skills [64]. This study also showed that the odds of good food hygiene practices were higher among food handlers who had formal education as compared to those who had not. Accordingly, the odds ratio of isolating Salmonella was 7.2 times (AOR: 7.2, 95\% CI 1.2-51.2) higher in butchers and restaurants where the workers did not attend any formal education compared to those who attended secondary school and above. In line with this finding, a study conducted by Asrat et al. [65] in Ethiopia also revealed the importance of education for food handlers to ensure food safety.

Wiping cloth was used for cleaning purposes particularly at butcher shops and restaurants in the current study, and the intention was good. However, it was reused the whole day and can accumulate microorganisms that can be transferred to the retailer operators' hands, to utensil surfaces, and finally to meat. Clothes have also been reported to be ineffective in removing microorganisms, thereby increasing the chance of cross-contamination [66].

There was improper disposal of leftover dirty materials (gastrointestinal content, horn, shank, and bones) collected from daily slaughtered animals at the abattoirs. The floor of the abattoir ought to be hard concrete and impenetrable, to minimize dirt and permit seepage and simplicity of cleaning. Likewise, a rooftop is critical to shield the carcass from the weather and to diminish the temperature in the abattoir [67]. In the present study, the floor of the abattoirs was made of concrete and impervious material but has no ceiling. Even though washing of the floor takes place every day at the end of the slaughtering process, the slaughter wall is not cleaned and washed at the end of the working day. Although different factors were there for cross-contamination of the meat, the training of the workers, the educational background of the meat handlers, and the food safety and hygiene knowledge of the meat handlers play a vital role.
The prevalence of Salmonella from raw meat in this study, though low, could give a cautionary signal for the conceivable event of food-borne diseases capable of causing outbreaks and pose a public health risk through the exported meat and meat products to the international market. The poor sanitation of butchers/restaurants, the poor hygiene of the slicing materials, the cutting boards, and the food handler's surfaces might have contributed to the reported prevalence of Salmonella in the current study. The absence of washing basins and first aid kits in butchers and restaurants might be attributed to the failure of local authorities to enforce existing food-related laws, lack of resources, and information about hygienic practices as the vast majority of the laborers had no formal training in sanitation. Poor individual cleanliness is one of the most significant sources of contamination for foods. Therefore, control measures along the meat processing chain, namely, abattoirs, meat processing plants, distributors, and consumers, should be undertaken to minimize the risk of cross-contamination and food-borne infections caused by Salmonella spp. [68].

\section{Conclusion}

The current study showed the highest prevalence of Salmonella isolates from butcher shops. The dominant serotype was S. typhimurium. The high rate of MDR Salmonella suggests a potential health risk to consumers from the consumption of raw meat. Education, knowledge of food safety, hygiene, and training on food safety and hygiene of workers are predictors of Salmonella isolation. The poor meat handling and poor personal hygienic practices of workers in the retail shops and restaurants may pose a risk of food-borne disease. Therefore, the use of soft, absorbent disposable paper towels for drying hands instead of cloth, continuous food safety education, and training for meat handlers are recommended to enhance good safety practices. There is a need to emphasize discouraging raw meat consumption and indiscriminate use of antimicrobial drugs. Besides, regular antimicrobial susceptibility surveillance is essential.

\section{Data Availability}

The datasets used and/or analyzed during the current research are available from the corresponding author on reasonable request.

\section{Ethical Approval}

The proposal was reviewed and approved by the research and an ethical review committee of Ambo University. Permission to conduct the study was also obtained from the towns' municipality with the letter written from Ambo University.

\section{Consent}

Informed oral consent was obtained from each study subject before they were asked to give information and meat 
samples, and they were informed that all the information and results of meat samples are only for the study.

\section{Conflicts of Interest}

The authors declare that they have no conflicts of interest.

\section{Authors' Contributions}

EZG conceived the idea, designed the project, supervised the work, analyzed and interpreted the data, and drafted the final manuscript for publication. GTB performed the laboratory tests and participated in drafting the article. $\mathrm{BMB}$, EJS, and HAA designed the project, supervised the work, and revised the manuscript. LMM and NDT participated in data collection, field and laboratory supervision, and gave comments on the manuscript. All authors approved the final version of the article for publication.

\section{Acknowledgments}

The authors would like to extend their appreciation to Ambo University for financially supporting the project. The authors are also grateful to the workers of the abattoirs, butchers, and restaurants in Ambo and Holeta towns. The authors are thankful to Dr. Roger P. Johnson, Dr. Linda Cole, Shaun Kernaghan, Ketna Mistry, Ann Perets, and Betty Wilkie of the Public Health Agency of Canada, National Microbiology Laboratory at Guelph for serotyping of the Salmonella isolates. This work was supported by Ambo University. The funder had no role in study design, data collection, and analysis, decision to publish, or preparation of the manuscript.

\section{References}

[1] M. L. Bartholomew, R. T. Heffernan, J. G. Wright et al., "Multistate outbreak of salmonella enterica serotype enteritidis infection associated with pet Guinea pigs," Vector-Borne and Zoonotic Diseases, vol. 14, no. 6, pp. 414-421, 2014.

[2] T. Beyene, H. Yibeltie, B Chebo et al., "Identification and antimicrobial susceptibility profile of Salmonella isolated from selected dairy farms, abattoir, and humans at Asella town, Ethiopia," Journal of Veterinary Science and Technology, vol. 7, no. 3, p. 320, 2016.

[3] S. F. Altekruse, D. L. Swerdlow, and S. J. Wells, "Factors in the emergence of food borne diseases," Veterinary Clinics of North America: Food Animal Practice, vol. 14, no. 1, pp. 1-15, 1998.

[4] T. Hald, "2 - pathogen update: Salmonella," in Advances in Microbial Food Safety, J. Sofos, Ed., pp. 25-46, Woodhead Publishing, Cambridge, UK, 2013.

[5] M. Y. Popoff and L. E. Le Minor, "Genus Salmonella," in Bergey's Manual of Systematic Bacteriology Part B, J. T. Staley, Ed., pp. 764-799, Springer, New York, NY, USA, 2nd edition, 2005.

[6] F. M. Sánchez-Vargas, M. A. Abu-El-Haija, and O. G. GómezDuarte, "Salmonella infections: an update on epidemiology, management, and prevention," Travel Medicine and Infectious Disease, vol. 9, no. 6, pp. 263-277, 2011.

[7] F. W. Brenner, R. G. Villar, F. J. Angulo, R. Tauxe, and B. Swaminathan, "Salmonella nomenclature," Journal of Clinical Microbiology, vol. 38, no. 7, pp. 2465-2467, 2000.
[8] R. Dieckmann and B. Malorny, "Rapid screening of epidemiologically important Salmonella enterica subsp. enterica serovars by whole-cell matrix-assisted laser desorption ionization-time of flight mass spectrometry," Applied and Environmental Microbiology, vol. 77, no. 12, pp. 4136-4146, 2011.

[9] S. E. Majowicz, J. Musto, E. Scallan et al., "The global burden of NontyphoidalSalmonellaGastroenteritis," Clinical Infectious Diseases, vol. 50, no. 6, pp. 882-889, 2010.

[10] S. Le Hello, R. S. Hendriksen, B. Doublet et al., "International spread of an epidemic population of Salmonella enterica serotype Kentucky ST198 resistant to ciprofloxacin," Journal of Infectious Diseases, vol. 204, no. 5, pp. 675-684, 2011.

[11] R. S. Hendriksen, A. R. Vieira, S. Karlsmose et al., "Global monitoring of Salmonella serovar distribution from the world health organization global foodborne infections network country data bank: results of quality assured laboratories from 2001 to 2007," Foodborne Pathogens and Disease, vol. 8, no. 8, pp. 887-900, 2011.

[12] Y. Kumar, A. Sharma, R. Sehgal, and S. Kumar, "Distribution trends of Salmonella serovars in India (2001-2005)," Transactions of the Royal Society of Tropical Medicine and Hygiene, vol. 103, no. 4, pp. 390-394, 2009.

[13] W. H. Organization, Risk Assessments of Salmonella in Eggs and Broiler Chickens, World Health Organization, Geneva, Switzerland, 2002.

[14] N. Mrema, S. Mpuchane, and B. A. Gashe, "Prevalence of Salmonella in raw minced meat, raw fresh sausages and raw burger patties from retail outlets in Gaborone, Botswana," Food Control, vol. 17, no. 3, pp. 207-212, 2006.

[15] G. Tadesse and E. Z. Gebremedhin, "Prevalence of Salmonella in raw animal products in Ethiopia: a meta-analysis," $B M C$ Research Notes, vol. 8, no. 1, p. 163, 2015.

[16] J. E. Stevenson, K. Gay, T. J. Barrett, F. Medalla, T. M. Chiller, and F. J. Angulo, "Increase in nalidixic acid resistance among non-typhi Salmonella enterica isolates in the United States from 1996 to 2003," Antimicrobial Agents and Chemotherapy, vol. 51, no. 1, pp. 195-197, 2007.

[17] D. Muleta and M. Ashenafi, "Salmonella, Shigella and growth potential of other food-borne pathogens in Ethiopian street vended foods," East African Medical Journal, vol. 78, no. 11, pp. 576-580, 2001.

[18] G. Girma, "Prevalence, antibiogram and growth potential of Salmonella and Shigella in Ethiopia: implications for public health: a review," Research Journal of Microbiology, vol. 10, no. 7, pp. 288-307, 2015.

[19] L. Garedew, Z. Hagos, Z. Addis, R. Tesfaye, and B. Zegeye, "Prevalence and antimicrobial susceptibility patterns of Salmonella isolates in association with hygienic status from butcher shops in Gondar town, Ethiopia," Antimicrobial Resistance \& Infection Control, vol. 4, p. 21, 2015.

[20] M. Thrusfield, Veterinary Epidemiology; Describing Disease Occurrence, Blackwell Publishing, Cambridge, UK, 3rd edition, 2005.

[21] ISO, Microbiology of Food and Animal Feeding Stuff -Preparation of Test Samples, Initial Suspension, and Decimal Dilutions for Microbiological Examination -- Part 2 Specific Rules for the Preparation of Meat and Meat Products, ISO, Geneva, Switzerland, 2003.

[22] CLSI: Clinical and Laboratory Standards Institute, Performance for Antimicrobial Disk Susceptibility Testing, CLSI, Wayne, PA, USA, 2017.

[23] C. R. Shipp and B. Rowe, "A mechanised microtechnique for salmonella serotyping," Journal of Clinical Pathology, vol. 33, no. 6, pp. 595-597, 1980. 
[24] P. A. Grimont and F.-X. Weill, "Antigenic formulae of the Salmonella serovars," WHO Collaborating Center for Reference and Research on Salmonella, vol. 9, pp. 1-166, 2007.

[25] C. Meyer, S. Thiel, U. Ullrich, and A. Stolle, "Salmonella in raw meat and by-products from pork and beef," Journal of Food Protection, vol. 73, no. 10, pp. 1780-1784, 2010.

[26] A. M. Lammerding, M. M. Garcia, E. D. Mann et al., "Prevalence of Salmonella and thermophilic Campylobacter in fresh pork, beef, veal and poultry in Canada1," Journal of Food Protection, vol. 51, no. 1, pp. 47-52, 1988.

[27] C. Zhao, B. Ge, J. De Villena et al., "Prevalence of Campylobacter spp., Escherichia coli, and Salmonella serovars in retail chicken, Turkey, pork, and beef from the greater Washington, D.C., area," Applied and Environmental Microbiology, vol. 67, no. 12, pp. 5431-5436, 2001.

[28] J. M. Bosilevac, M. N. Guerini, N. Kalchayanand, and M. Koohmaraie, "Prevalence and characterization of Salmonellae in commercial ground beef in the United States," Applied and Environmental Microbiology, vol. 75, no. 7, pp. 1892-1900, 2009.

[29] T. Y. Thung, S. Radu, N. A. Mahyudin et al., "Prevalence, virulence genes and antimicrobial resistance profiles of Salmonella serovars from retail beef in Selangor, Malaysia," Frontiers in Microbiology, vol. 8, 2018.

[30] B. Yang, D. Qu, X. Zhang et al., "Prevalence and characterization of Salmonella serovars in retail meats of marketplace in Shaanxi, China," International Journal of Food Microbiology, vol. 141, no. 1-2, pp. 63-72, 2010.

[31] A. A. Moawad, H. Hotzel, O Awad et al., "Occurrence of Salmonella enterica and Escherichia coli in raw chicken and beef meat in northern Egypt and dissemination of their antibiotic resistance markers," Gut Pathogens, vol. 9, p. 57, 2017.

[32] A. Kebede, J. Kemal, H. Alemayehu, and S. Habte Mariam, "Isolation, identification, and antibiotic susceptibility testing of Salmonella from slaughtered bovines and ovines in Addis Ababa Abattoir Enterprise, Ethiopia: a Cross-Sectional Study," International Journal of Bacteriology, vol. 2016, Article ID 3714785, 2016.

[33] K. Kore, B. Asrade, K. Demissie, and K. Aragaw, "Characterization of Salmonella isolated from apparently healthy slaughtered cattle and retail beef in Hawassa, southern Ethiopia," Preventive Veterinary Medicine, vol. 147, pp. 11-16, 2017.

[34] A. Aklilu, D. Kahase, M. Dessalegn et al., "Prevalence of intestinal parasites, salmonella and shigella among apparently health food handlers of Addis Ababa University student's cafeteria, Addis Ababa, Ethiopia," BMC Research Notes, vol. 8, no. 1, p. 17, 2015.

[35] H. Tassew, A. Abdissa, G. Beyene, and S. Gebre-Selassie, "Microbial flora and food borne pathogens on minced meat and their susceptibility to antimicrobial agents," Ethiopian Journal of Health Sciences, vol. 20, no. 3, pp. 137-143, 2010.

[36] T. Eguale, W. A. Gebreyes, D. Asrat, H. Alemayehu, J. S. Gunn, and E. Engidawork, "Non-typhoidal Salmonella serotypes, antimicrobial resistance, and co-infection with parasites among patients with diarrhea and other gastrointestinal complaints in Addis Ababa, Ethiopia," BMC Infectious Diseases, vol. 15, no. 1, p. 497, 2015.

[37] T. Lamboro, T. Ketema, and K. Bacha, "Prevalence and antimicrobial resistance in Salmonella and Shigella species isolated from outpatients, Jimma university specialized hospital, southwest Ethiopia," Canadian Journal of Infectious Diseases and Medical Microbiology, vol. 2016, Article ID 4210760, 2016.
[38] A. Hiko, H. Irsigler, G. Ameni, K.-H. Zessin, and R. Fries, "Salmonella serovars along two beef chains in Ethiopia," The Journal of Infection in Developing Countries, vol. 10, no. 11, pp. 1168-1176, 2016.

[39] W. Wabeto, Y. Abraham, and A. A. Anjulo, "Detection and identification of antimicrobial-resistant Salmonella in raw beef at Wolaita Sodo municipal abattoir, Southern Ethiopia," Journal of Health, Population and Nutrition, vol. 36, no. 1, p. 52, 2017.

[40] L. Ketema, Z. Ketema, B Kiflu et al., "Prevalence and antimicrobial susceptibility profile of Salmonella serovars isolated from slaughtered cattle in Addis Ababa, Ethiopia," BioMed Research International, vol. 2018, Article ID 9794869, 2018.

[41] S. Alemu and B. M. Zewde, "Prevalence and antimicrobial resistance profiles of Salmonella enterica serovars isolated from slaughtered cattle in Bahir Dar, Ethiopia," Tropical Animal Health and Production, vol. 44, no. 3, pp. 595-600, 2012.

[42] G. Ameya, T. Tsalla, F. Getu, and E. Getu, “Antimicrobial susceptibility pattern, and associated factors of Salmonella and Shigella infections among under-five children in Arba Minch, South Ethiopia," Annals of Clinical Microbiology and Antimicrobials, vol. 17, no. 1, p. 1, 2018.

[43] M. Ejo, L. Garedew, Z. Alebachew, and W. Worku, "Prevalence and antimicrobial resistance of Salmonella isolated from animal-origin food items in gondar, Ethiopia," BioMed Research International, vol. 2016, Article ID 4290506, 2016.

[44] A. Dabassa and K. Bacha, "The prevalence and antibiogram of Salmonella and Shigella isolated from an abattoir, Jimma town, South west Ethiopia," International Journal of Pharma and Bio Sciences, vol. 3, no. 4, pp. 143-148, 2012.

[45] R. Li, J. Lai, Y. Wang et al., "Prevalence and characterization of Salmonella species isolated from pigs, ducks and chickens in Sichuan Province, China," International Journal of Food Microbiology, vol. 163, no. 1, pp. 14-18, 2013.

[46] C. K. Okoro, R. A. Kingsley, T. R. Connor et al., "Intracontinental spread of human invasive Salmonella Typhimurium pathovariants in sub-Saharan Africa," Nature Genetics, vol. 44, no. 11, pp. 1215-1221, 2012.

[47] D. Alemayehu, B. Molla, and A. Muckle, "Prevalence and antimicrobial resistance pattern of Salmonella isolated from apparently healthy slaughtered cattle in Ethiopia," Tropical Animal Health and Production, vol. 35, no. 4, pp. 309-319, 2003.

[48] B. L. Arruda, E. R. Burrough, and K. J. Schwartz, "Salmonella enterica I 4,[5],12:i:- associated with lesions typical of swine enteric salmonellosis," Emerging Infectious Diseases, vol. 25, no. 7, pp. 1377-1379, 2019.

[49] CDC, Antibiotic Resistance Threats in the United States of America, CDC, Atlanta, GA, USA, 2013.

[50] M. Mama and G. Alemu, "Prevalence, antimicrobial susceptibility patterns and associated risk factors of Shigella and Salmonella among food handlers in Arba Minch University, South Ethiopia," BMC Infectious Diseases, vol. 16, no. 1, p. 686, 2016.

[51] F. Abunna, D. Ashenafi, T. Beyene, D. Ayana, B. Mamo, and R. Duguma, "Isolation, identification and antimicrobial susceptibility profiles of Salmonella isolates from dairy farms in and around Modjo town, Ethiopia," Ethiopian Veterinary Journal, vol. 21, no. 2, pp. 92-108, 2017.

[52] Z. Addis, N. Kebede, Z. Worku, H. Gezahegn, A. Yirsaw, and T. Kassa, "Prevalence and antimicrobial resistance of Salmonella isolated from lactating cows and in contact humans 
in dairy farms of Addis Ababa: a cross-sectional study," $B M C$ Infectious Diseases, vol. 11, p. 222, 2011.

[53] R. D. Abdi, F. Mengstie, A. F Beyi et al., "Determination of the sources and antimicrobial resistance patterns of Salmonella isolated from the poultry industry in Southern Ethiopia," BMC Infectious Diseases, vol. 17, no. 1, p. 352, 2017.

[54] D. Marami, K. Hailu, and M. Tolera, "Prevalence and antimicrobial susceptibility pattern of Salmonella and Shigella species among asymptomatic food handlers working in Haramaya University cafeterias, Eastern Ethiopia," BMC Research Notes, vol. 11, no. 1, p. 74, 2018.

[55] A. Molloy, S. Nair, F. J. Cooke et al., "First report ofSalmonella entericaSerotype paratyphi A azithromycin resistance leading to treatment failure," Journal of Clinical Microbiology, vol. 48, no. 12, p. 4655, 2010.

[56] A. Terfassa and M. Jida, "Prevalence and antibiotics susceptibility pattern of Salmonella and Shigella species among diarrheal patients attending Nekemte referral hospital, Oromia, Ethiopia," International Journal of Microbiology, vol. 2018, Article ID 9214689, 2018.

[57] G. Beyene, S. Nair, D. Asrat, Y. Mengistu, H. Engers, and J. Wain, "Multidrug resistant Salmonella Concord is a major cause of salmonellosis in children in Ethiopia," The Journal of Infection in Developing Countries, vol. 5, no. 01, pp. 23-33, 2011.

[58] DACAE, "Standard treatment guidelines for district hospitals," in Addis Ababa Ethiopia: Drug Administration and Control Authority of EthiopiaDACAE, Addis Ababa, Ethiopia, 2004.

[59] J. A. Crump, M. Sjölund-Karlsson, M. A. Gordon, and C. M. Parry, "Epidemiology, clinical presentation, laboratory diagnosis, antimicrobial resistance, and antimicrobial management of invasive Salmonella infections," Clinical Microbiology Reviews, vol. 28, no. 4, pp. 901-937, 2015.

[60] DACA, Antimicrobials Use Resistance and Containment Baseline Survey Syntheses of Findings, DACA, Addis Ababa, Ethiopia, 2009.

[61] N. H. Gebrekirstos, B. D. Workneh, Y. S Gebregiorgis et al., "Non-prescribed antimicrobial use and associated factors among customers in drug retail outlet in Central Zone of Tigray, northern Ethiopia: a cross-sectional study," Antimicrobial Resistance \& Infection Control, vol. 6, no. 1, p. 70, 2017.

[62] C. Jianu and I. Goleţ, "Knowledge of food safety and hygiene and personal hygiene practices among meat handlers operating in western Romania," Food Control, vol. 42, pp. 214-219, 2014.

[63] N.-A. Abdul-Mutalib, M.-F. Abdul-Rashid, S. Mustafa, S. Amin-Nordin, R. A. Hamat, and M. Osman, "Knowledge, attitude and practices regarding food hygiene and sanitation of food handlers in Kuala Pilah, Malaysia," Food Control, vol. 27, no. 2, pp. 289-293, 2012.

[64] S.-H. Park, T.-K. Kwak, and H.-J. Chang, "Evaluation of the food safety training for food handlers in restaurant operations," Nutrition Research and Practice, vol. 4, no. 1, pp. 58-68, 2010.

[65] S. Asrat, K. M. Davis, and R. R. Isberg, "Modulation of the host innate immune and inflammatory response by translocated bacterial proteins," Cellular Microbiology, vol. 17, no. 6, pp. 785-795, 2015.

[66] M. Fawzi, N. F. Gomaa, and W. M. Bakr, "Assessment of handwashing facilities, personal hygiene and the bacteriological quality of hand washes in some grocery and dairy shops in Alexandria, Egypt," J Egypt Public Health Assoc, vol. 84, no. 1-2, pp. 71-93, 2009.
[67] WHO, Food Hygiene Basic Texts, WHO, Rome, Italy, 2009.

[68] FAO, Interventions for the Control of Nontyphoidal Salmonella Spp. in Beef and Pork, FAO, Rome, Italy, 2015. 